\title{
The role of pancreatic polypeptide in pancreatic diseases
}

\author{
Mariola Śliwińska-Mossoń1, A-E, Grzegorz Marek ${ }^{2, ~(-E}$, Halina Milnerowicz ${ }^{1, A, E, F}$ \\ ${ }^{1}$ Department of Biomedical and Environmental Analyses, Wroclaw Medical University, Poland \\ ${ }^{2}$ Second Department and Clinic of General and Oncological Surgery, Wroclaw Medical University, Poland \\ A - research concept and design; $\mathrm{B}$ - collection and/or assembly of data; $\mathrm{C}$ - data analysis and interpretation; \\ $D$ - writing the article; $E$ - critical revision of the article; $F$ - final approval of the article
}

Address for correspondence

Mariola Śliwińska-Mossoń

E-mail: mariola.sliwinska-mosson@umed.wroc.pl

Funding sources

None declared

\section{Conflict of interest}

None declared

Received on April 12, 2016

Reviewed on August 13, 2016

Accepted on September 7, 2016

\begin{abstract}
The aim of this study was to review the diagnostic significance of pancreatic polypeptide (PP) in pancreatic diseases. PP may play a significant role in monitoring the development of the disease and the patient's healing process, particularly after the removal of a portion of the pancreas. Determining PP in acute pancreatitis is quite controversial. At the ${ }^{\text {st }}$ stage of severe pancreatic damage, there is excessive PP release followed by its fall. In patients with chronic pancreatitis, a significant decrease in PP secretion was found in the presence of a food stimulant. In this case, PP could be a good marker for determining the stage of pancreatitis. Pancreatic polypeptide also functions as a hepatic glucose regulator. PP increases hepatic insulin sensitivity, resulting in reduced hepatic glucose production. Therefore, impaired hepatic insulin sensitivity in chronic pancreatitis is abrogated after the PP administration. Endocrine pancreatic tumors initially grow without specific symptoms. In contrast, they are almost always correlated with elevated serum pancreatic polypeptide. Therefore, the level of PP may be a good diagnostic parameter confirming the presence of pancreatic cancer. Depending on the type of disease, the polypeptide concentration can be increased or decreased, evidencing the disease progress or regression.
\end{abstract}

Key words: pancreatic polypeptide, acute pancreatitis, chronic pancreatitis, diabetes

D0I

10.17219/acem/65094

\section{Copyright}

Copyright by Author(s)

This is an article distributed under the terms of the

Creative Commons Attribution Non-Commercial License

(http://creativecommons.org/licenses/by-nc-nd/4.0/) 


\section{Introduction}

The clinical significance of pancreatic polypeptide (PP) is closely related to the function of this hormone in the body. Due to its localization in the pancreas, it appears to be a useful diagnostic parameter in acute and chronic pancreatitis (AP, CP).,

Playing its role in the body, PP reduces energy demand, thereby decreasing the amount of food intake. This observation contributed to the development of research on the treatment of obesity. ${ }^{3}$ Moreover, studies using the immunohistochemical technique showed an increase in the number and change of PP cell location in the pancreatic parenchymal tissue sections obtained from tobacco smoking patients with chronic pancreatitis and concomitant diabetes. Long lasting CP may lead to the development of insulin-dependent diabetes. PP determination can be a useful diagnostic parameter of diabetes development in the course of chronic pancreatitis. ${ }^{1,2}$ The aim of the review was to provide the current stage of knowledge on PP and its function in the body, in physiological and pathological conditions of the pancreas.

\section{Discussion}

\section{Pancreatic polypeptide}

Pancreatic polypeptide is a hormone consisting of 36 amino acids with a molecular weight of approx. $4227 \mathrm{Da}$. Its precursor is a protein composed of $95 \mathrm{ami}$ no acid residues called pancreatic prepropolipeptyde. The C-terminus of pancreatic polypeptide is tyrosine. ${ }^{4,5}$ PP belongs to the peptide family, which includes neuropeptide Y (NPY) and peptide YY (PYY). Five different affinity subtypes of NPY-, PYY- and PP-binding receptors were identified. ${ }^{5-8}$ They include receptors Y1, Y2, Y4, Y5, and Y6. Pancreatic polypeptide has the greatest affinity with the Y4 receptor, which is a heptahelical receptor that binds the G-protein, thereby inhibiting the action of adenylatecyclase and influencing the phospholipase $\mathrm{C}$ activation. ${ }^{7}$ In human plasma, $\mathrm{PP}$ is represented in at least 4 forms: 1-36PP, 3-36PP, the other 2 forms have not been fully described yet. The identification was performed using the HPLC chromatography technique. ${ }^{9} \mathrm{PP}$ circulates in the blood mainly as a dimer. PP half-life in vivo is 6-7 $\mathrm{min}$, and it is excreted in the active form predominantly by the kidneys. The normal PP concentration in human plasma ranges from 40 to $80 \mathrm{pmol} / \mathrm{L}$. PP release from cells is a rapid process, and in the case of certain mammals and humans, this phenomenon is biphasic. A certain role in the secretion of PP into the bloodstream is played by bloating intestines, cholecystokinin release (to a lesser extent, also other intestinal hormones) as well as direct digestible nutrients. However, the primary incentive taking part in PP cell stimulation to secrete this hormone is the release of acetylcholine from cholinergic fibers extending to the pancreas. Hypoglycemia, during which the muscarinic acetylcholine receptors and, to a lesser extent, adrenergic receptors are activated, also has an influence on PP release. ${ }^{10} \mathrm{PP}$ is released into the plasma during after-meal stimulation, in particular in the case of meals containing protein and fat. Its physiological role includes gastric stimulation inhibition, gastric acid secretion stimulation, delayed gastric emptying (Fig. 1). This hormone inhibits the exocrine pancreatic secretion and insulin secretion in the organ, as well as stimulates the contraction of the gallbladder. These effects slow down the digestive process and the process of absorption of nutrients into circulation, preventing the glucose concentration in the blood from increasing after a meal. ${ }^{8} \mathrm{PP}$ secretion remains at a low level when fasting but its concentration increases at all stages of digestion. PP secretion depends on the part of the gastrointestinal tract. A strong stimulus affecting PP release in the cephalic phase decreases the concentration of blood glucose and hypoglycemia-induced insulin. A key role in the mechanism of PP release is played by an intact vagus nerve and the cholinergic nerves. Vagotomy and muscarinic receptor antagonist treatment inhibit PP secretion. ${ }^{8,11}$

\section{The significance of pancreatic polypeptide in obesity}

Previous studies have shown that PP appears to be involved in regulating food intake and energy balance. ${ }^{8,11}$ Transgenic mice overexpressing PP gained less weight because of decreased food intake and this was accompanied by decreased fat mass. Studies on the impact of peripheral PP action on food intake were also carried out on mice with the obesity gene. PP concentration was observed in the plasma of healthy and obese mice. No increase in the hormone levels in the plasma of obese subjects was observed, despite increased PP content in the pancreas of tested animals. These results indicate that PP cell hyperplasia occurs in obese mice due to lower PP levels in the plasma and because of reduced tissue sensitivity to the hormone. ${ }^{12}$ The congenital human obesity (PraderWilli syndrome) is characterized, similarly as in the case of experimental animals, by PP cell hyperplasia, obesity, hyperglycemia and hyperinsulinemia. The problem of obesity in this case has its source in the lack of adequate PP secretion stimulation. Intravenous PP infusion in obese mice leads to increased secretion of this polypeptide and reduction of food intake by tested objects. These observations suggest that PP circulating in the blood stream affects feeding behavior. PP administered to obese mice by injection twice a day for 2 weeks reduces their weight growth. PP can modulate the vagovagal reflexes as well as the autonomous regulation of gastrointestinal function. The hormone changes the function of these neurons, leading in effect to decreased food intake. 


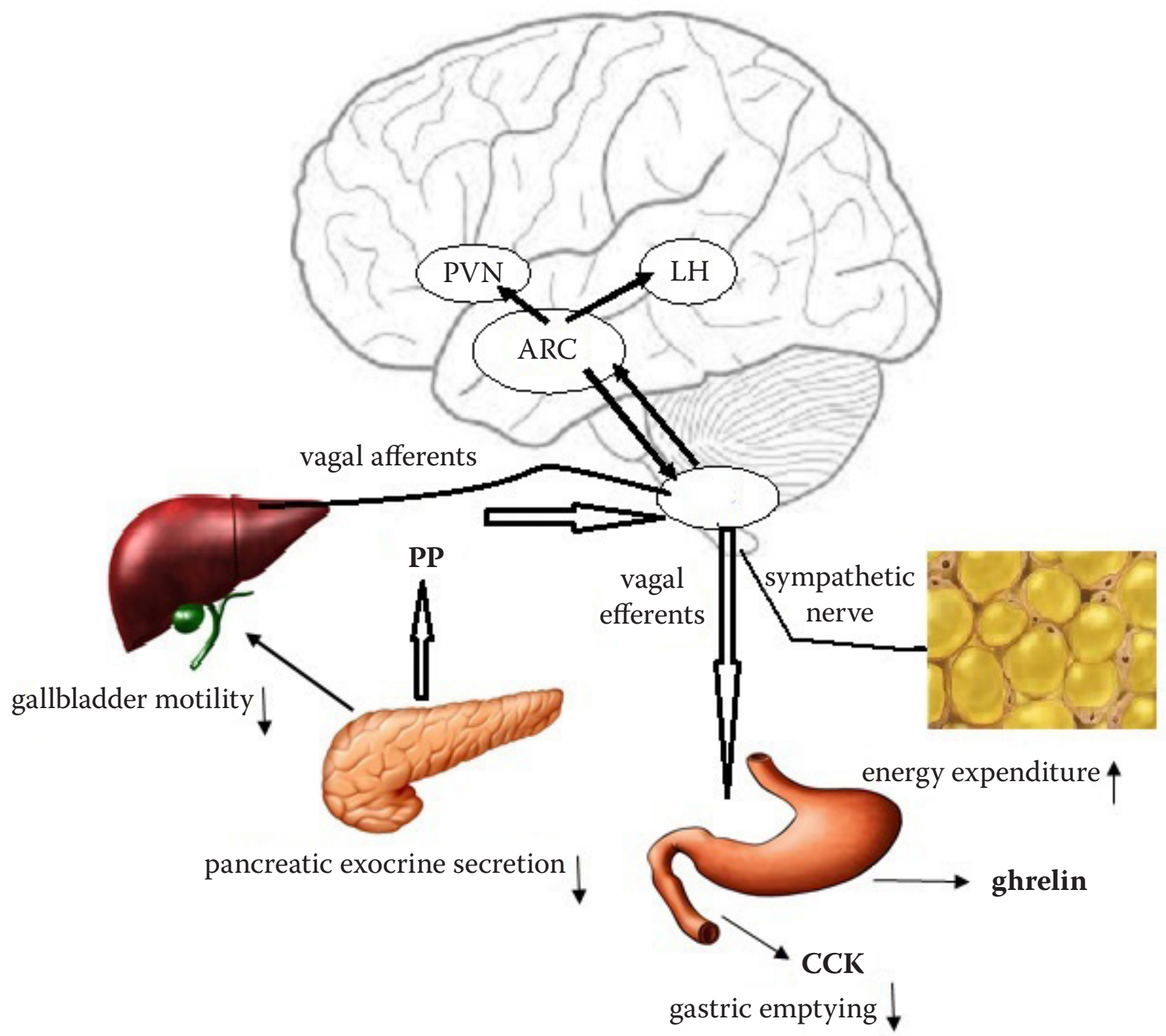

Fig. 1. Regulatory functions of pancreatic polypeptide: inhibition of pancreatic exocrine secretion, gallbladder motility and gastric emptying

PP acts through blood circulation and vagal afferents, and the signal is transmitted to the dorsal vagal complex (DVC) and the hypothalamus (ARC). PP decreases food intake partly through stimulation of cholecystokinin (CKK) and inhibition of ghrelin, and increases energy expenditure through stimulation of the sympathetic nervous system innervating brown adipose tissue. ARC - arcuate nucleus of the hypothalamus; $\mathrm{LH}-$ lateral hypothalamic area; PVN - paraventricular nucleus of the hypothalamus.?

PP secreted by food stimulation becomes a signal to inhibit further eating and participates in the control of meal sizes by changing gastric emptying during meals. ${ }^{11,13}$

Intravenous peripheral administration of PP to genetically obese ob/ob mice induced a state of negative energy balance because of decreased food intake and increased energy expenditure. ${ }^{14}$

Recent studies have shown that the satiety effects of PP involve an impact on the Y4 receptors in hypothalamic nuclei, which affects the pathway distinct from the one mediating the functions of PYY. ${ }^{12,15}$ Research suggests that pancreatic polypeptide, the preferential Y4 receptor agonist, regulates the energy balance of the body. When administered intravenously or intraperitoneally into mice, it accelerates the metabolism rate, reducing at the same time hyperglycemia, insulin resistance and hyperlipidemia in obese mice. The 20-fold higher concentration of the hor- mone in the plasma of transgenic mice, when compared to healthy subjects, reduces food intake and, consequently, body weight and the adipose tissue content in these rodents. ${ }^{16} \mathrm{PP}$ impacts food intake reduction via the Y4 receptor localized in the brainstem and the hypothalamus. Recent work suggests that PP is also the primary ligand for the $\mathrm{Y} 6$ receptors, which also regulate energy homeostasis. ${ }^{17}$

The above PP effect is abolished when the vagus nerve has been cut in rodents. This observation suggests that PP has an impact on the process of food intake inhibition by the vagus nerve. It was noted that PP might also affect the process of food intake stimulation. This phenomenon is dependent on the peripheral control of PP release. Differences in the impact of PP on feeding behavior may result from the stimulation of different receptors. However, this phenomenon requires further studies to investigate specific mechanisms. There is a decrease in serum 
PP concentration after meals, and it is proportionate to the increasing body weight. Other studies have shown elevated PP levels after meals in patients with anorexia. ${ }^{5}$ In the case of patients with Prader-Willi syndrome, it was noted that basic PP release was reduced increasingly in relation to older age and larger body weight. 3,13

PP inhibitory effect on the feeding process was demonstrated in a number of experimental studies. In the case of mice with acute and chronic pancreatitis, a decrease in the food intake was observed. Obese mice with leptin deficiency were noted to lose weight, improve insulin resistance and reduce hyperlipidemia after intraperitoneal PP administration. Furthermore, transgenic mice with elevated serum PP levels had reduced body weight and showed a decreased demand for food when compared to wild type strains. ${ }^{16}$ Intravenous PP administration helps maintain normal weight in the case of human subjects by reducing food intake by $25 \%$ in $24 \mathrm{~h} .{ }^{18}$ Furthermore, the administration of suitable PP doses 2 times a day to patients with Prader-Willi syndrome caused a 12\% lower demand for food. ${ }^{18}$ Low serum levels of PP have been observed in obese people and intravenous infusions of PP reduced food intake, and PP secretion was thought to be primarily under vagal control, although other factors have also been shown to alter serum PP concentrations. ${ }^{12,19-21}$

\section{The importance of pancreatic polypeptide in acute and chronic pancreatitis}

Both acute pancreatitis and chronic pancreatitis lead to the disturbance of endocrine and exocrine secretions of the organ. High incidence of these diseases leads to severe complications, and researchers are seeking diagnostic markers allowing early detection. One of the useful parameters in diagnosing pancreatic disease appears to be pancreatic polypeptide.

In most cases, acute pancreatitis is a fairly mild and self-limiting process. The morphology of this disease corresponds to the inflammatory type interstitial edema. The mild form of acute pancreatitis is associated with mild disorders in other organs. AP may have the necrohemorrhagic form (5-20\% of cases), in the course of which an uncontrolled development of inflammation may appear. The pathomechanism of the disease consists in the premature activation of trypsinogen by intracellular lysosomal enzymes in the granules of pancreatic lobules of secretory cells, leading to the destruction of the organ. ${ }^{22}$ The diagnostic role of PP in patients with acute pancreatitis is not clearly identified. In animal experiments, there was a significant PP concentration level increase in the first $6 \mathrm{~h}$ after acute pancreatitis onset. ${ }^{23}$ In another experiment, it was demonstrated that PP concentration rises significantly during the first $96 \mathrm{~h}$ after the onset of acute hemorrhagic pancreatitis.

In a pilot study conducted on patients with acute pancreatitis, an increase in PP concentration in the plasma was noted; however, subsequent studies did not confirm the correlation. The observed value of the average hormone concentration in the plasma of patients with mild to moderate acute pancreatitis was similar to the mean PP concentration in the plasma of healthy subjects. ${ }^{24}$

In clinical conditions, this AP marker has diagnostic value when its concentration is and remains elevated for a longer time during the disease. In experimental studies on an animal model, PP concentration measurement satisfies the diagnostic marker criteria, while in studies on human subjects, this correlation does not occur. Therefore, it was concluded that the PP measurement during fasting did not have diagnostic significance in the course of acute pancreatitis. Differences in the studies may be explained by the fact that in the case of animal experiments, PP measurements started upon acute pancreatitis onset, while in the case of human subjects, the hormone measurements, even after $72 \mathrm{~h}$ following the onset of disease symptoms, were still considered to be done in the initial phase of the disease. One should also take into account the acute pancreatitis pathophysiology in experimental and clinical cases. It is not fully explained yet to what extent the experimental models reflect acute pancreatitis in humans. Most studies did not differentiate between mild, moderate and severe disease types. ${ }^{1}$

Chronic pancreatitis is a heterogeneous syndrome of clinical symptoms, which include: recurrent, prolonged hypogastric pain, anorexia, vomiting, nausea, fatty food intolerance, persistent diarrhea, foul-smelling stool, and progressive weight loss. In the morphological image of the pancreas, there are prevailing focal changes or diffuse necrosis of the glandular tissue with segmental or diffuse fibrosis, the latter being a progressive and irreversible process. As the disease progresses, exocrine and endocrine insufficiency of the organ develops. ${ }^{22}$

Several years ago, it was found in multiple studies that the determination of PP concentrations in serum, together with immunoreactive trypsin and isoamylase activity, may be useful in chronic pancreatitis diagnosis. ${ }^{1}$ To determine particular disease subtypes from mild, through moderate to severe, PP concentration was measured in patients with chronic pancreatitis. ${ }^{25}$ During the research, lower PP concentration and greatly reduced secretion of the hormone after appropriate stimulation were noted. In addition, other studies have shown that alcohol-abusing patients with chronic pancreatitis have a low fasting PP concentration. The disadvantage of using PP level assessment in the diagnosis of chronic pancreatitis is a significant impact of age on the hormone level in the fasting state. Some researchers believe that PP level assessment has little diagnostic value in the case of patients with mild to moderate chronic pancreatitis, due to its low sensitivity. ${ }^{1}$ It was found that other hormone secretion (meal, pentagastrin, cholecystokinin) is an important element when assessing the PP level in patients with chronic pancreatitis. In order to clarify 
the role of PP in the course of chronic pancreatitis, a prospective study should be performed on a healthy control group and on a group of patients with other gastrointestinal disorders. ${ }^{1}$ It is well documented that a persistently reduced PP level in the course of CP corresponds to the degree of pancreatic islet damage, and is also an indication of exocrine and endocrine insufficiency of the pancreas (Fig. 2). ${ }^{26}$

\section{The significance of PP in diabetes associated with chronic pancreatitis}

Pancreatic diabetes may also be associated with a deficiency of pancreatic islet cell hormones: insulin, glucagon, and PP. PP cells are located at the periphery of Langerhans islets and are distributed between the vesicles and mucous membrane of the pancreatic duct epithelium (Fig. 2). It is probable that insulin-producing beta cells located in the "center" of the pancreatic islets are protected against damage by surrounding PP cells. ${ }^{26,27}$ Accordingly, decreased PP production may be the first sign of cell dysfunction in the islets of the pancreas. Important clinical manifestations of hormonal disturbances occur late in the course of diabetes. Therefore, there is a role of PP as an early marker of hormonal insufficiency. PP may be used as a prognostic factor for chronic pancreatitis. ${ }^{1}$ In another group of studies, it was confirmed that the absence of the beta-cell function in the course of chronic pancreatitis is associated with significant PP cell function impairment and severe exocrine pancreatic insufficiency. ${ }^{28}$ The role of PP in the regulation of insulin secretion after pancreatitis is not fully clarified because changes in glucose tolerance are associated with abnormal PP levels. The assumption that PP functions as a hormone influencing the glucose level in blood has already been made. ${ }^{26,29}$ However, in the animal model, acute PP infusion failed to improve glucose tolerance and did not augment insulin release, but there was an effect of PP on hepatic sensitivity to insulin by decreasing hepatic glucose production. ${ }^{29}$

Impaired hepatic insulin sensitivity in chronic pancreatitis was observed after PP administration. Therefore, the hepatic insulin resistance could be a reversible effect of PP deficiency as well as of reduced stimulation of PP receptors on hepatocytes. These processes can be PP-specific, but there is a possibility that, due to high pancreatic polypeptide homology with the Y and PYY neuropeptides, the latter are involved in the modulating action of insulin in the course of hepatic glucose release. It is suggested that PP has a key role in glucose homeostasis. Furthermore, it can serve as an early diagnostic and prognostic marker for chronic pancreatitis in diabetes. ${ }^{1}$

PP infusions were also able to improve glycemic profiles both in patients and in animals with chronic pancreatitis. ${ }^{30}$ Adult patients with type 1 diabetes appear to have elevated levels of pancreatic polypeptide. This suggests
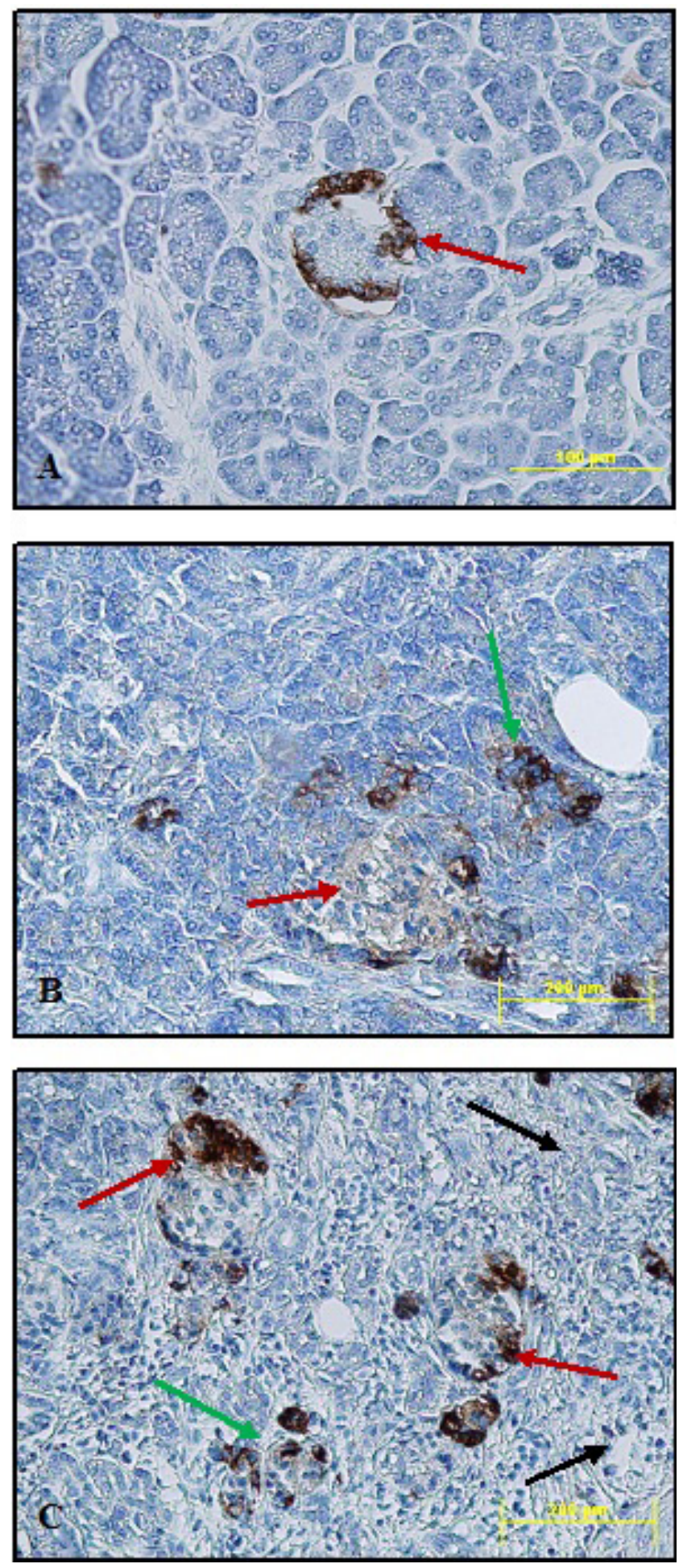

Fig. 2. Immunohistochemical localization of pancreatic polypeptide in the tissue of the pancreas

A - normal pancreas (a strong immunohistochemical reaction in a single cell of PP at the periphery islets of Langerhans); B - chronic pancreatitis (a weak immunohistochemical reaction for PP in the cells at the periphery islets of Langerhans, a moderate to strong immunohistochemical reaction for $\mathrm{PP}$ in cells between acinar cells); C - chronic pancreatitis with diabetes (a very strong immunohistochemical reaction in cells of PP in the whole area of islets of Langerhans, a strong immunohistochemical reaction PP in a single cell secreting pancreatic polypeptide in the part of the exocrine pancreas, reduction of stromal tissue in the process of fibrosis). Arrows: red - pancreatic islets; green - cells PP between acinar cells; black - fibrous lesions in the sublayer. Photograph - own study. 
an association between serum PP levels and insulin sensitivity. ${ }^{31}$ The exact mechanism of their action is currently under debate.

PP treatment led to increased insulin secretion from isolated human islets, most likely because it decreased the secretion of somatostatin, a known inhibitor of insulin secretion. Kim et al. present evidence for the functional significance of increased PP secretion within pancreatic islets. It decreases somatostatin secretion, which has an inhibitory effect on insulin secretion. ${ }^{7}$ Elevated PP levels in type 2 diabetes are likely to represent a compensatory response in an effort to restore normal glycemic level by decreasing somatostatin secretion within islets. ${ }^{32}$ Gutislet interactions have been well studied because incretin hormones (GIP, GLP-1), released from scattered enteric endocrine cells, regulate glucose-mediated insulin secretion. Somatostatin inhibits the release of many gastrointestinal endocrine hormones but the levels of all these actually increase in the circulation after eating. Hence, the suppression of somatostatin postcranially in the gut is a vital necessity. Gut-islet interactions via hormones are bidirectional. ${ }^{7}$ Additionally, somatostatin is known to inhibit pancreatic exocrine secretion, hepatic bile secretion and gallbladder emptying. ${ }^{7,30,33}$ These 3 effects need to be disinhibited after eating. PP seems to be responsible for via local inhibition of somatostatin secretion. ${ }^{7}$

The immunohistochemical technique allows for the identification of morphological abnormalities in the Langerhans islet cells in the case of patients with diabetes. Regularly, PP cells are arranged at the periphery of islets. The percentage and the distribution of PP cells increases significantly during the course of diabetes. ${ }^{26}$

Researchers from the University of Prague conducted a study on the level of hormones secreted into the gastrointestinal tract in young adults with type 1 diabetes. The highlighted group of metabolically active hormones secreted by pancreatic islet cells comprised of: amylin, ghrelin, GLP-1, GIP, leptin, PYY polypeptide, insulin, and pancreatic polypeptide. Studies have shown a consistent correlation of the measured levels of concentration of these peptide hormones with glycemia, with control metabolic markers as well as anthropometric measurements of patients. Serum PP levels showed no statistically significant difference between the study and control groups. The value of PP was $139.00 \pm 96.38 \mathrm{pg} / \mathrm{mL}$ with the median $111.18 \mathrm{pg} / \mathrm{mL} .{ }^{31}$ No data concerning PP levels in patients with type 1 diabetes are available in the international literature; however, the presence of PP cell hyperplasia in young adults with type 1 diabetes has been documented. PP concentrations in the study group chosen by the researchers showed some upwards tendency, yet they are not statistically significant. The elevated PP level in patients with type 1 diabetes is associated with hyperglycemia. At the same time, PP increases hepatic insulin sensitivity and reduces hepatic glucose production. ${ }^{31,34}$ The study showed the PP action in a positive relation with amylin and a negative correlation with insulin. The researchers suggest a positive role of PP as a drug medication for patients with type I diabetes. ${ }^{31}$

A group of scientists from the Michigan and Minnesota Universities conducted a study on the secretion of glucagon, catecholamines and PP in patients diagnosed with type 1 diabetes, after pancreatic transplantation. The stimulus which triggered PP release was the supply of insulin, which induced a state of hypoglycemia, and stimulation with arginine. The study group consisted of 38 patients with diabetes, after an organ transplant, as well as 54 patients with diabetes, without a transplant. The control group consisted of 26 non-diabetic healthy patients. No significant differences in PP secretion between the test groups and the control group were found. However, PP secretion in both groups of patients with diabetes was lower compared to the control group. The researchers suggest that similar PP secretion results among patients from the control group can be found only in patients with native pancreas. It is suggested that after a series of PP monitoring studies such patients may provide valuable information concerning pancreatic transplants in the future. ${ }^{32} \mathrm{PP}$ might thus become an important glucose metabolism marker. ${ }^{34,35}$

In type 2 diabetic subjects, PP cells secrete excess of PP and plasma PP levels are significantly elevated in the postprandial state, compared to non-diabetic subjects. ${ }^{32}$ Loss of pancreatic parenchyma following the resection of this organ causes extreme disturbances in the glucose homeostasis, similar to those in pancreatic diabetes. This form of glucose intolerance is different from other forms of diabetes owing to more frequent episodes of iatrogenic hypoglycemia. The improvement of postoperative care and a possibility of an early disease diagnosis greatly extend the length and quality of life of patients after pancreatic resection. The pathophysiology of pancreatic diabetes is associated with the deficiency of pancreatic hormones and changes in response of the liver and other organs to lower than normal hormone levels. PP deficiency is associated with hyperglycemia resulting from an unrestrained hepatic glucose production. It is a characteristic symptom of diabetes caused by pancreatic resection. Influencing PP release may mitigate the difficulties with glucose concentrations. PP may probably also improve liver cell sensitivity to insulin, thus beneficially influencing the glycemic control system. ${ }^{36}$

\section{Pancreatic polypeptide as a tumor marker}

The first case of a tumor secreting only PP (PP-oma) by Bordi was registered in 1978. The pancreatic tumor was accidentally discovered during surgery of a duodenal ulcer. Immunohistochemical studies of the tumor showed a very large amount of PP immunoreactive cells. The serum concentration in these patients was an important indicator that this patient had a 10 times higher concentration 
of PP in the blood. In addition to the excessive secretion of the peptide, there were no clinical and laboratory signs of tumor development. ${ }^{37}$ PP-oma gives some atypical symptoms, e.g., abdominal pain, itchy rash, weight loss and watery diarrhea. ${ }^{38}$

Pancreatic polypeptide secreting tumors are usually benign adenomas. ${ }^{37,38}$ They can also transform into malignant tumors with metastatic potential to adjacent lymph nodes and the liver, which can be potentially fatal. However, most PP-omas are benign tumors with a prevalent location in the pancreatic head. The recommended treatment for these patients is surgical removal of the tumor. In some cases, a total pancreatectomy with lymphadenectomy is required. After this treatment the level of PP in the blood must be monitored due to the possible PP cell hyperplasia. ${ }^{37,38}$

Pancreatic polypeptide-producing cells in small amounts often occur in endocrine tumors of the pancreas. Most commonly they occur in glucagonomas, but also in ininsulinomas, gastrinomas, and VIP-omas. In these tumors, immunoreactive PP cells may be discretely scattered throughout the tissue or form focal aggregates. ${ }^{37}$ In patients with endocrine tumors of the pancreas, the serum PP level is considerably increased compared to the corresponding age control group. This parameter can only be a confirmation of the diagnosis, since the correct concentration of PP does not exclude the presence of pancreatic tumors. Therefore, a preoperative measurement of the hormone level has little clinical value. On the other hand, PP level is a valid parameter for monitoring the course of the disease. The increase in the concentration of the basic PP after a resection may be the result of incomplete removal of the tumor cells. This may also be indicative of PP cell hyperplasia, but not of their cancer growth. ${ }^{38}$

In patients with multiple endocrine neoplasia type 1 (MEN1), there are 2 characteristic changes. The first is the microadenomatosis of islet cells of the pancreas, while the other, less frequent changes take the form of large single endocrine tumors. Numerous adenomas are scattered along the entire pancreatic gland. The number of microadenomas may exceed 100, and the diameter of a single adenoma can sometimes be greater than $0.5 \mathrm{~cm}$. These changes are functionally asymptomatic and considered benign, both clinically and histologically. Large tumors are sporadic in MEN1 and occur most frequently as background microadenomatosis of islet cells. Most likely, MEN1 gene inactivation (chromosome 11q13) is responsible for the development of pancreatic endocrine tumors secreting pancreatic polypeptide. ${ }^{37,39}$

Mutch et al. attempted to define the relationship between the level of fasting PP and the presence of radiographically detectable pancreatic endocrine tumors in patients with MEN1. The results indicated a greatly increased concentration of pancreatic polypeptide (PP levels above 3 times higher compared to the corresponding age control group). These values were $95 \%$ sensitive and $88 \%$ specific for islet cell tumors detected radiologically. The relationship, however, serves as a screening test because normal PP concentration does not exclude the presence of a tumor. ${ }^{39}$ Standardization of the test, in which a stimulus that stimulates the secretion of PP is food, allows for the confirmation of pancreatic endocrine tumors in $75 \%$ of young patients with MEN1, in which the tumor is still asymptomatic. ${ }^{37}$ In turn, the test in which the stimulus stimulates the secretion of pancreatic polypeptide secretin does not exhibit the diagnostic sensitivity. Elevated concentrations of PP in patients with MEN1 syndrome is clinically very important because it is almost always associated with the presence of pancreatic islet cell tumor, which can be surgically removed. After the removal of the tumor, the level of PP can be a good prognostic indicator for monitoring patients. ${ }^{39}$ Excessive secretion of PP is also observed in endocrine tumors localized in the gastrointestinal tract and related organs. PP cell growth was also seen in tumors of the thyroid, lungs and ovaries.

However, non-pancreatic tumors secreting pancreatic polypeptide are rare. It was found that pancreatic polypeptide and related peptides with PP were found in $62 \%$ of cases of carcinoid rectum. Rectal carcinoid, secreting pancreatic polypeptide, behaves similarly to other peptide secreting tumors. Usually, they take the form of small polyps located in the submucosa, revealing a benign character. When the tumor diameter exceeds $2 \mathrm{~cm}$, it tends toward malignancy. Therefore, it may metastasize to the lymph and blood, placing itself in the wall of the intestine, liver and lymph nodes. ${ }^{37}$

\section{Conclusions}

Pancreatic polypeptide is a hormone secreted by PP cells, located at the periphery of the pancreatic Langerhans islets. The physiological roles of this hormone include what follows: stomach stimulation inhibition, delayed gastric emptying, pancreatic exocrine function inhibition, and inhibition of insulin secretion and hepatic glucose production. As part of its role in the functioning of the body, PP reduces the demand for energy, thereby decreasing the amount of food intake. This observation has led to the development of research concerning the treatment of obesity. It turned out that PP regulates the body energy balance. An intravenous or intraperitoneal PP administration decreased metabolic rate in obese mice. In addition, PP resulted in the animal body weight loss, hyperlipidemia and insulin resistance. The clinical significance of PP can also be observed in the course of acute and chronic pancreatitis. In acute pancreatitis, due to the rapid progression of pancreatic islet destruction, PP concentration is initially high, but then it decreases. The dynamics of changes in hormone concentrations may be an indication of the disease phase. However, in the course of CP, PP concentration is maintained 
at a low level. It is proportional to the degree of islet damage and points to exocrine and endocrine secretion failure of the pancreas. Long lasting chronic pancreatitis may develop into insulin-dependent diabetes. PP cells located at the periphery of the Langerhans islets play a protective role with regard to the beta cells located in the islet center. Therefore, reduced PP levels may be the first signal of pancreatic endocrine cell failure. In addition, PP increases hepatic insulin sensitivity by reducing hepatic glucose production. It has been suggested that PP plays a role in the inflammation process of the pancreas; therefore, determining the PP concentration in the serum may be a useful diagnostic parameter of the diabetes development in the course of chronic pancreatitis.

Elevated concentrations of pancreatic polypeptide are often observed in patients with endocrine tumors of the pancreas. Almost always there is a high level of PP in patients with MEN1. However, in these conditions high levels of PP can only confirm the diagnosis because normal concentration of PP does not exclude the presence of a tumor. On the other hand, the level of PP can be a good parameter to monitor diagnostic patient after the removal of the tumor. The increase in the basic concentration of PP after the operation may be the result of incomplete removal of the tumor cells. It may also indicate a PP cell hyperplasia.

\section{References}

1. Hennig R, Kekis PB, Friess $H$, Adrian TE, Büchler MW. Pancreatic polypeptide in pancreatitis. Peptides. 2002;23:331-338.

2. Śliwińska-Mossoń $M$, Milnerowicz $H$, Milnerowicz $S$, Nowak $M$, Rabczyński J. Immunohistochemical localization of somatostatin and pancreatic polypeptide in smokers with chronic pancreatitis. Acta Histochem. 2012;5:495-502.

3. Suzuki K, Simpson KA, Minnion JS, Shillito JC, Bloom SR. The role of gut hormones and the hypothalamus in appetite regulation. Endocr J. 2010;57:359-372.

4. Piao FL, Yuan K, Bai GY, Han JH, Park WH, Kim SH. Different regulation of atrial ANP release through neuropeptide Y2 and Y4 receptors. J Korean Med Sci. 2008;23:1027-1032.

5. Śliwińska-Mossoń M, Borowiecka K, Milnerowicz H. Neuropeptides Y, YY, PP and their clinical significance. Postepy Hig Med Dosw. 2013;67:631-636.

6. Lundell I, Blomqvist AG, Berglund MM, et al. Cloning of a human receptor of the NPY receptor family with high affinity for pancreatic polypeptide and peptide YY. J Biol Chem. 1995;270:29123-29128.

7. Kim W, Fiori JL, Shin YK, et al. Pancreatic polypeptide inhibits somatostatin secretion. FEBS Lett. 2014;588:3233-3239.

8. Kojima S, Ueno N, Asakawa A, et al. A role for pancreatic polypeptide in feeding and body weight regulation. Peptides. 2007;28:459-463.

9. Gingerich RL, Akpan JO, Leith KM, Gilbert WR. Patterns of immunoreactive pancreatic polypeptide in human plasma. Regul Pept. 1991;33:275-285.

10. Hazelwood RL. The pancreatic polypeptide (PP-Fold) family: Gastrointestinal, vascular, and feeding behavioral implications. Proc Soc Exp Biol Med. 1993;202:44-63.

11. Katsuura G, Asakawa A, Inui A. Roles of pancreatic polypeptide in regulation of food intake. Peptides. 2002;23:323-329.

12. Ueno $N$, Inui $A$, Iwamoto $M$, et al. Decreased food intake and body weight in pancreatic polypeptide-overexpressing mice. Gastroenterology. 1999;117:1427-1432.

13. Berntson GG, Zipf WB, O'Dorisio TM, Hoffman JA, Chance RE. Pancreatic polypeptide infusions reduce food intake in Prader-Willi syndrome. Peptides. 1993;14:497-503.
14. Asakawa A, Inui A, Yuzuriha $H$, et al. Characterization of the effects of pancreatic polypeptide in the regulation of energy balance. Gastroenterology. 2003;124:1325-1336.

15. Shi YC, Lin Z, Lau J, et al. PYY3-36 and pancreatic polypeptide reduce food intake in an additive manner via distinct hypothalamic dependent pathways in mice. Obesity. 2013;12:669-678.

16. Sainsbury A, Bergen HT, Boey D, et al. Y2Y4 receptor double knockout protects against obesity due to a high-fat diet or $\mathrm{Y} 1$ receptor deficiency in mice. Diabetes. 2006;55:19-26.

17. Yulyaningsih E, Loh K, Lin S, et al. Pancreatic polypeptide controls energy homeostasis via Npy6r signaling in the suprachiasmatic nucleus in mice. Cell Metab. 2014;19:58-72.

18. Batterham RL, le Roux CW, Cohen MA, et al. Pancreatic polypeptide reduces appetite and food intake in humans. $J$ Clin Endocrinol Metab. 2003;88:3989-3992.

19. Glaser B, Zoghlin G, Pienta K, Vinik Al. Pancreatic polypeptide response to secretin in obesity: Effects of glucose intolerance. Horm Metab Res. 1988;20:288-292.

20. Taylor IL, Impicciatore M, Carter DC, Walsh JH. Effect of atropine and vagotomy on pancreatic polypeptide response to a meal in dogs. Am J Physiol. 1978;235:443-447.

21. Linnestad $P$, Schrumpf E. Pancreatic polypeptide release stimulated by food, secretin and cholecystokinin in chronic pancreatitis. Scand J Gastroenterol. 1983;18:385-389.

22. Dembińska-Kieć A, Naskalski JW. Laboratory Diagnosis of Clinical Biochemistry Elements. $3^{\text {rd }}$ ed. Wrocław: Urban \& Partner; 2010:744-747.

23. Pappas TN, Yovos JG, Ellison EC, et al. Pancreatic polypeptide in acute pancreatitis and small-bowel infarction in dogs. Dig Dis Sci. 1981;26:1013-1018.

24. Dominguez-Munoz JE, Pieramico $O$, Büchler $M$, Malfertheiner $P$. Exocrine pancreatic function in the early phase of human acute pancreatitis. Scand J Gastroenterol. 1995;30:186-191.

25. Matsumoto $M$, Wakasugi $H$, Ibayashi H. Plasma human pancreatic polypeptide response in chronic pancreatitis. Gastroenterol Jpn. 1982;17:25-30.

26. Śliwińska-Mossoń M, Milnerowicz H. Distribution of pancreatic polypeptide-secreting endocrine cells in nondiabetic and diabetic cases. Appl Immunohistochem Mol Morphol. 2016. [Epub ahead of print]

27. Valenzuela JE, Taylor IL, Walsh JH. Pancreatic polypeptide response in patients with chronic pancreatitis. Dig Dis Sci. 1979;24:862-864.

28. Larsen S, Hilsted J, Tronier B, Worning H. Pancreatic hormone secretion in chronic pancreatitis without residual $\beta$-cell function. Acta Endocrinol (Copenh). 1988;118:357-364.

29. Bastidas JA, Couse NF, Yeo CJ, et al. The effect of pancreatic polypeptide infusion on glucose tolerance and insulin response in longitudinally studied pancreatitis-induced diabetes. Surgery. 1990;107:661-668.

30. Harris AG. Somatostatin and somatostatin analogues: Pharmacokinetics and pharmacodynamic effects. Gut. 1994;35(Suppl 3):1-4.

31. Huml M, Kobr J, Siala K, et al. Gut peptide hormones and pediatric type 1 diabetes mellitus. Physiol Res. 2011;60:647-658.

32. Floyd JC Jr, Fajans SS, Pek S, Chance RE. A newly recognized pancreatic polypeptide: Plasma levels in health and disease. Recent Prog Horm Res. 1976;33:519-570.

33. Śliwińska-Mossoń M, Veselý M, Milnerowicz H. The clinical significance of somatostatin in pancreatic diseases. Ann Endocrinol (Paris). 2014;75:232-240.

34. Brunicardi FC, Chaiken RL, Ryan AS. Pancreatic polypeptide administration improves abnormal glucose metabolism in patients with chronic pancreatitis. J Clin Endocrinol Metab. 1996;81:3566-3572.

35. Diem P, Redmon BR, Abid M, et al. Glucagon, catecholamine and pancreatic polypeptide secretion in type I diabetic recipients of pancreas allografts. J Clin Invest. 1990;86:2008-2013.

36. Maeda $\mathrm{H}$, Hanazaki H. Pancreatogenic diabetes after pancreatic resection. Pancreatology. 2011;11:268-276.

37. Bordi C, Azzoni C, D'Adda T, Pizzi S. Pancreatic polypeptide-related tumors. Peptides. 2002;23:339-348.

38. Bellows C, Hague S, Jaffe B. Pancreatic polypeptide islet cell tumor: Case report and review of the literature. J Gastrointest Surg. 1998;2:526-532. 
39. Mutch MG, Frisella MM, DeBenedetti MK, et al. Pancreatic polypeptide is a useful plasma marker for radiographically evident pancreatic islet cell tumors in patients with multiple endocrine neoplasia type 1. Surgery. 1997;122:1012-1020. 